\title{
An Augmented Electric Field Integral Equation for Layered Medium Green's Function
}

\author{
Yongpin P. Chen, Student Member, IEEE, Lijun Jiang, Member, IEEE, Zhi-Guo Qian, Member, IEEE, and \\ Weng Cho Chew, Fellow, IEEE
}

\begin{abstract}
This paper proposes an augmented electric field integral equation (A-EFIE) for layered medium Green's function. The newly developed matrix-friendly formulation of layered medium Green's function is applied in this method. By separating charge as extra unknown list, and enforcing the current continuity equation, the traditional EFIE can be cast into a generalized saddlepoint system. Frequency scaling for the matrix-friendly formulation is analyzed when frequency tends to zero. Rank deficiency and the charge neutrality enforcement of the A-EFIE for layered medium Green's function is discussed in detail. The electrostatic limit of the A-EFIE is also analyzed. Without any topological loopsearching algorithm, electrically small conducting structures embedded in a general layered medium can be simulated by using this new A-EFIE formulation. Several numerical results are presented to validate this method at the end of this paper.
\end{abstract}

Index Terms-Augmented electric field integral equation, dyadic Green's function for layered medium, low frequency.

\section{INTRODUCTION}

C OMPUTATIONAL electromagnetics becomes indispensable as a CAD methodology in various electrical engineering applications, such as in integrated circuit and wireless communication device. The operating frequency of the electrical systems keeps on increasing to several gigahertz, meanwhile fabrication process has achieved nanoscale. Hence, a broadband simulation tool is badly needed for capturing circuit physics of the tiny structures as well as wave physics for the whole package. Unfortunately, the commonly used electric field integral equation (EFIE) method solved by the method of moments (MoM) [1] with the Rao-Wilton-Glisson (RWG) basis function [2] suffers from a "low frequency breakdown" problem, where vector potential gradually looses its significance compared with the scalar potential part when the frequency decreases, and the EFIE operator becomes singular [3]. Various approaches have been proposed to overcome this

\footnotetext{
Manuscript received July 09, 2010; accepted September 15, 2010. Date of publication December 30, 2010; date of current version March 02, 2011. This work was supported by HKG GRF Grants 711508, 711609 (General Research Fund).

Y. P. Chen and L. Jiang are with the Department of Electrical and Electronic Engineering, University of Hong Kong, China.

Z.-G. Qian is with Intel Corporation, Chandler, AZ 85226 USA.

W. C. Chew is with the Department of Electrical and Electronic Engineering, University of Hong Kong, China on leave from the Department of Electrical and Computer Engineering, University of Illinois at Urbana-Champaign, Urbana, IL 61801 USA (e-mail: wcchew@hku.hk).

Color versions of one or more of the figures in this paper are available online at http://ieeexplore.ieee.org.

Digital Object Identifier 10.1109/TAP.2010.2103042
}

problem in the last few years. One of the most popular remedies is the loop-tree or loop-star decomposition [4], [5], where the solenoidal and irrotational components of the unknown current can be separated due to the quasi-Helmholtz decomposition (also known as Hodge decomposition), to capture inductance physics and capacitance physics when the frequency tends to zero. However, even after frequency normalization, the matrix is still ill-conditioned. Preconditioning is necessary to improve the convergence when iterative solvers are applied. Several effective preconditioners have been proposed, either based on the basis-rearrangement, where the favorable property of electrostatic problems is utilized [6], or based on the near-field interactions, where the incomplete factorization with a heuristic drop strategy is applied [7]. By using the Calderón identity and the dual basis or Buffa-Christiansen basis function [8], [9], a more effective preconditioner has been constructed [10]-[12]. The loop-tree or loop-star method has also been implemented with the layered medium Green's function [13]-[16], which is more versatile in the simulation of printed antenna and planarly integrated circuit [17]-[19].

However, one big issue associated with the loop-tree or loopstar method is the loop-search process. It is a bottleneck for complicated interconnecting geometries with increasing number of unknowns, where many entangle global loops may exist. Situation becomes even worse when layered medium with conducting ground plane is involved, where extra implicit global loops are introduced because of the vias. To avoid the loopsearch process, the idea of separating current and charge to construct a stable formulation has been studied recently. The current and charge integral equation (CCIE) method [20] puts charges into the extra unknown list and manipulates the equation system to be of the second kind. While in the separated potential integral equation (SPIE) method [21], the scalar potential is included as the unknowns, where resistive loss and dielectric loss are introduced to flatten the condition number when the frequency is low. In the recent developed augmented electric field integral equation (A-EFIE) method [22], [23], the similar idea of separating current and charge as independent unknowns is applied. By enforcing the current continuity equation explicitly and implementing a proper frequency scaling, the EFIE can be cast into a generalized saddle point system [24]. With the help of a constraint preconditioner for the saddle point system and the mixed-form fast multipole algorithm, a real package problem with more than one million unknowns has been successfully solved on a personal computer [23].

In this paper, we extend the A-EFIE method into the layered medium problem, to make it applicable for simulating 
structures embedded in a general layered substrate. The matrix-friendly layered medium Green's function [15], [16] is applied to achieve the A-EFIE formulation. First, the A-EFIE formulation for free space Green's function is reviewed. Then, the frequency scaling is analyzed for both lossless and lossy media and the corresponding A-EFIE is set up for layered medium. Next, the rank deficiency and the necessity of enforcing charge neutrality condition is discussed in detail in the context of layered medium. After that, the electrostatic limit of the A-EFIE is analyzed to show the consistency with the static solver. Finally, several numerical examples are presented to validate this method.

\section{The Augmented EFIE Formula for Free Space GREEN'S FUNCTION}

For a conducting object illuminated by an external excitation, electric current will be induced on the surface. This current will then generate an electric field that exactly cancels the incident electric field inside the object and will yield a scattered field in the outside region due to the extinction theorem [3]. The electric field integral equation (EFIE) can then be derived as

$$
\hat{t} \cdot \mathcal{L}\left(\mathbf{r}, \mathbf{r}^{\prime}\right) \mathbf{J}\left(\mathbf{r}^{\prime}\right)=-\hat{t} \cdot \mathbf{E}^{i}(\mathbf{r})
$$

where the operator $\mathcal{L}$ connects the source current and the scattered field via the free space dyadic Green's function

$$
\begin{aligned}
\mathcal{L}\left(\mathbf{r}, \mathbf{r}^{\prime}\right) \mathbf{J}\left(\mathbf{r}^{\prime}\right) & =i \omega \mu_{0}\left\langle\overline{\mathbf{G}}\left(\mathbf{r}, \mathbf{r}^{\prime}\right), \mathbf{J}\left(\mathbf{r}^{\prime}\right)\right\rangle \\
& =i \omega \mu_{0} \int d \mathbf{r}^{\prime} \overline{\mathbf{G}}\left(\mathbf{r}, \mathbf{r}^{\prime}\right) \cdot \mathbf{J}\left(\mathbf{r}^{\prime}\right) \\
\overline{\mathbf{G}}\left(\mathbf{r}, \mathbf{r}^{\prime}\right) & =\left(\overline{\mathbf{I}}+\frac{\nabla \nabla}{k_{0}^{2}}\right) g\left(\mathbf{r}, \mathbf{r}^{\prime}\right)
\end{aligned}
$$

here the scalar Green's function $g\left(\mathbf{r}, \mathbf{r}^{\prime}\right)$ is the solution to the scalar Helmholtz wave equation with a point source

$$
g\left(\mathbf{r}, \mathbf{r}^{\prime}\right)=\frac{e^{i k_{0}\left|\mathbf{r}-\mathbf{r}^{\prime}\right|}}{4 \pi\left|\mathbf{r}-\mathbf{r}^{\prime}\right|}
$$

To solve the EFIE in (1), the induced current $\mathbf{J}\left(\mathbf{r}^{\prime}\right)$ can be expanded by using the RWG basis function [2] defined on an adjacent triangular patch pair

$$
\mathbf{f}_{i}(\mathbf{r})= \begin{cases}\frac{\boldsymbol{\rho}_{i}^{+}}{2 A_{i}^{+}}, & \mathbf{r} \in T_{i}^{+} \\ -\frac{\boldsymbol{\rho}_{i}^{-}}{2 A_{i}^{-}}, & \mathbf{r} \in T_{i}^{-} \\ 0, & \text { otherwise }\end{cases}
$$

where $A_{i}^{ \pm}$is the area of the two triangles associated with the $i$ th basis, and the $\boldsymbol{\rho}_{i}^{ \pm}$is the vector pointing to a point $\mathbf{r}$ from the two vertices. Here, the function is normalized by its edge length for convenience. The surface divergence can then be written as

$$
\nabla_{s} \cdot \mathbf{f}_{i}(\mathbf{r})= \begin{cases}\frac{1}{A_{i}^{+}} & \mathbf{r} \in T_{i}^{+} \\ -\frac{1}{A_{i}^{-}} & \mathbf{r} \in T_{i}^{-} \\ 0, & \text { otherwise. }\end{cases}
$$

After applying the Galerkin procedure [1], the EFIE can be converted into a matrix system

$$
\overline{\mathbf{Z}} \cdot \mathbf{I}=\mathbf{V}
$$

with

$$
\overline{\mathbf{Z}}=i k_{0} \eta_{0} \overline{\mathbf{A}}+\frac{\eta_{0}}{i k_{0}} \overline{\mathbf{S}}
$$

here $\eta_{0}$ is the free space wave impedance and $k_{0}$ is the wave number. The $\mathbf{A}$ and $\mathbf{S}$ correspond to the magnetic vector potential and electric scalar potential

$$
\begin{aligned}
{[\overline{\mathbf{A}}]_{j i} } & =\left\langle\mathbf{f}_{j}(\mathbf{r}), g\left(\mathbf{r}, \mathbf{r}^{\prime}\right), \mathbf{f}_{i}\left(r^{\prime}\right)\right\rangle \\
{[\overline{\mathbf{S}}]_{j i} } & =\left\langle\nabla \cdot \mathbf{f}_{j}(\mathbf{r}), g\left(\mathbf{r}, \mathbf{r}^{\prime}\right), \nabla^{\prime} \cdot \mathbf{f}_{i}\left(\mathbf{r}^{\prime}\right)\right\rangle .
\end{aligned}
$$

From (8)-(10), we can see that the vector potential block and the scalar potential one are imbalanced when the frequency is low, namely $k_{0} \rightarrow 0$, since they are in different frequency order. The operator $\mathcal{L}$ becomes singular because any divergence-free current is a solution to the EFIE in the quasi-static limit,

$$
\overline{\mathbf{S}} \cdot \mathbf{I} \simeq 0 .
$$

To balance the system, the charge can be separated and added into the unknown list to make the system stable in an augmented fashion [22], [23]. We define the normalized pulse basis function on each triangular patch as

$$
p_{i}(\mathbf{r})=\left\{\begin{array}{ll}
\frac{1}{A_{i}} & \mathbf{r} \in T_{i} \\
0, & \text { otherwise }
\end{array} .\right.
$$

If further defining a patch-to-patch scalar potential matrix as

$$
[\overline{\mathbf{P}}]_{j i}=\left\langle p_{j}(\mathbf{r}), g\left(\mathbf{r}, \mathbf{r}^{\prime}\right), p_{i}\left(\mathbf{r}^{\prime}\right)\right\rangle
$$

we can obtain the relationship between the patch-pair based (in terms of divergence of RWG basis) scalar potential matrix and the patch-based one

$$
\overline{\mathbf{S}}=\overline{\mathbf{D}}^{T} \cdot \overline{\mathbf{P}} \cdot \overline{\mathbf{D}}
$$

where the incidence matrix $\overline{\mathbf{D}}$ relates the domain of the RWG basis and the patch basis,

$$
[\overline{\mathbf{D}}]_{j i}= \begin{cases}1, & \text { Patch } j \text { is the positive part of RWG } i \\ -1, & \text { Patch } j \text { is the negative part of RWG } i \\ 0, & \text { otherwise. }\end{cases}
$$

Due to the current continuity equation, we have

$$
\overline{\mathbf{D}} \cdot \mathbf{J}=i k_{0} c_{0} \boldsymbol{\rho}
$$

where $c_{0}$ is the light speed in vacuum and $\boldsymbol{\rho}$ is the charge unknowns. Substituting the above equations into the EFIE matrix 
equation, and enforcing the current continuity equation explicitly, we can arrive at the following augmented-EFIE (A-EFIE) system

$$
\left[\begin{array}{cc}
\overline{\mathbf{A}} & \overline{\mathbf{D}}^{T} \cdot \overline{\mathbf{P}} \\
\overline{\mathbf{D}} & k_{0}^{2} \overline{\mathbf{I}}
\end{array}\right] \cdot\left[\begin{array}{c}
i k_{0} \mathbf{J} \\
c_{0} \boldsymbol{\rho}
\end{array}\right]=\left[\begin{array}{c}
\eta_{0}^{-1} \mathbf{V} \\
\mathbf{0}
\end{array}\right]
$$

This equation is the generalized saddle point system with the lower right block nearly equals to zero and various methods can be applied to solve this problem efficiently [24].

\section{The AUGMENTEd EFIE Formula FOR LAYERED MEDIUM GREEN'S FUNCTION}

\section{A. The Dyadic Green's Function for Layered Medium}

In a planarly layered medium, the dyadic Green's function has no closed-form spatial domain solution, it can only be expressed as an infinite integral (Sommerfeld integral). Various approaches have been developed to derive the layered medium Green's function, for instance, transmission line analog [13], Hertz potential approach [14], $E_{z}-H_{z}$ formulation [25], and vector wave formula [26]. Here, we apply the last one since it can be cast into a matrix-friendly form [15], [16], which has the singularity with the lowest order. The dyadic Green's function takes the form of

$$
\begin{aligned}
\overline{\mathbf{G}}\left(\mathbf{r}, \mathbf{r}^{\prime}\right) & =(\nabla \times \hat{z})\left(\nabla^{\prime} \times \hat{z}\right) g^{T E}\left(\mathbf{r}, \mathbf{r}^{\prime}\right) \\
& +\frac{1}{k_{n m}^{2}}(\nabla \times \nabla \times \hat{z})\left(\nabla^{\prime} \times \nabla^{\prime} \times \hat{z}\right) g^{T M}\left(\mathbf{r}, \mathbf{r}^{\prime}\right)
\end{aligned}
$$

where $k_{n m}^{2}=\omega^{2} \epsilon_{n} \mu_{m}, m$ is the index of the layer where source triangle resides and $n$ for observation layer. The $g^{\alpha}\left(\mathbf{r}, \mathbf{r}^{\prime}\right)$ is expressed as a Sommerfeld integral,

$$
g^{\alpha}\left(\mathbf{r}, \mathbf{r}^{\prime}\right)=\frac{i}{4 \pi} \int_{0}^{+\infty} \frac{d k_{\rho}}{k_{m z} k_{\rho}} J_{0}\left(k_{\rho} \rho\right) F^{\alpha}\left(k_{\rho}, z, z^{\prime}\right)
$$

where $F^{\alpha}\left(k_{\rho}, z, z^{\prime}\right)$ is the propagation factor [26] in $z$ direction for a given $k_{\rho}$, and $\alpha$ represents TE or TM polarization. In moment method implementation, the matrix-friendly formula is much more preferred, where the dyadic Green's function is divided into pieces and incorporated into the matrix elements

$$
\overline{\mathbf{Z}}=i \omega \mu_{m}\left\{\overline{\mathbf{Z}}^{s s}+\overline{\mathbf{Z}}^{z z}+\overline{\mathbf{Z}}^{z_{1}}+\overline{\mathbf{Z}}^{z_{2}}+\overline{\mathbf{Z}}^{\phi}\right\}
$$

where

$$
\begin{aligned}
{\left[\overline{\mathbf{Z}}^{s s}\right]_{j i} } & =\left\langle\mathbf{f}_{j s}(\mathbf{r}), g_{s s}\left(\mathbf{r}, \mathbf{r}^{\prime}\right), \mathbf{f}_{i s}\left(\mathbf{r}^{\prime}\right)\right\rangle \\
{\left[\overline{\mathbf{Z}}^{z z}\right]_{j i} } & =\left\langle\hat{z} \cdot \mathbf{f}_{j}(\mathbf{r}), g_{z z}\left(\mathbf{r}, \mathbf{r}^{\prime}\right), \hat{z} \cdot \mathbf{f}_{i}\left(\mathbf{r}^{\prime}\right)\right\rangle \\
{\left[\overline{\mathbf{Z}}^{z_{1}}\right]_{j i} } & =-\left\langle\hat{z} \cdot \mathbf{f}_{j}(\mathbf{r}), g_{z_{1}}\left(\mathbf{r}, \mathbf{r}^{\prime}\right), \nabla^{\prime} \cdot \mathbf{f}_{i}\left(\mathbf{r}^{\prime}\right)\right\rangle \\
{\left[\overline{\mathbf{Z}}^{z_{2}}\right]_{j i} } & =-\left\langle\nabla \cdot \mathbf{f}_{j}(\mathbf{r}), g_{z_{2}}\left(\mathbf{r}, \mathbf{r}^{\prime}\right), \hat{z} \cdot \mathbf{f}_{i}\left(\mathbf{r}^{\prime}\right)\right\rangle \\
{\left[\overline{\mathbf{Z}}^{\phi}\right]_{j i} } & =\left\langle\nabla \cdot \mathbf{f}_{j}(\mathbf{r}), g_{\phi}\left(\mathbf{r}, \mathbf{r}^{\prime}\right), \nabla^{\prime} \cdot \mathbf{f}_{i}\left(\mathbf{r}^{\prime}\right)\right\rangle
\end{aligned}
$$

with the Green's function components as

$$
g_{s s}\left(\mathbf{r}, \mathbf{r}^{\prime}\right)=k_{\rho}^{2} g^{T E}\left(\mathbf{r}, \mathbf{r}^{\prime}\right)
$$

$$
\begin{aligned}
& g_{z z}\left(\mathbf{r}, \mathbf{r}^{\prime}\right)=k_{m n}^{2} g^{T M}\left(\mathbf{r}, \mathbf{r}^{\prime}\right)-\partial_{z} \partial_{z^{\prime}} g^{T E}\left(\mathbf{r}, \mathbf{r}^{\prime}\right) \\
& g_{z_{1}}\left(\mathbf{r}, \mathbf{r}^{\prime}\right)=\frac{\mu_{n}}{\mu_{m}} \partial_{z^{\prime}} g^{T M}\left(\mathbf{r}, \mathbf{r}^{\prime}\right)+\partial_{z} g^{T E}\left(\mathbf{r}, \mathbf{r}^{\prime}\right) \\
& g_{z_{2}}\left(\mathbf{r}, \mathbf{r}^{\prime}\right)=\frac{\varepsilon_{m}}{\varepsilon_{n}} \partial_{z} g^{T M}\left(\mathbf{r}, \mathbf{r}^{\prime}\right)+\partial_{z^{\prime}} g^{T E}\left(\mathbf{r}, \mathbf{r}^{\prime}\right) \\
& g_{\phi}\left(\mathbf{r}, \mathbf{r}^{\prime}\right)=\frac{\partial_{z} \partial_{z^{\prime}}}{k_{n m}^{2}} g^{T M}\left(\mathbf{r}, \mathbf{r}^{\prime}\right)-g^{T E}\left(\mathbf{r}, \mathbf{r}^{\prime}\right) .
\end{aligned}
$$

\section{B. Frequency Scaling}

Since the primary (direct) term can be analyzed in the similar way as in free space, only secondary (reflected or transmitted) terms are considered in this section. We first assume that the layered medium is lossless. For general case, namely $\epsilon_{i} \neq \epsilon_{j}$ and $\mu_{i} \neq \mu_{j}$, when $\omega \rightarrow 0, k_{i z} \rightarrow i k_{\rho}$, the frequency scaling of the Fresnel reflection coefficient is

$$
R_{i, j}=\frac{p_{j} k_{i z}-p_{i} k_{j z}}{p_{j} k_{i z}+p_{i} k_{j z}}=\frac{p_{j}-p_{i}}{p_{j}+p_{i}} \sim O\left(\omega^{0}\right)
$$

where $p=\mu$ for TE wave and $p=\epsilon$ for TM wave. Then we can get the frequency scaling for other quantities:

$$
\tilde{R}_{i, j} \sim O\left(\omega^{0}\right), \tilde{M}_{m} \sim O\left(\omega^{0}\right), \tilde{T}_{m n} \sim O\left(\omega^{0}\right)
$$

where $\tilde{R}, \tilde{M}$ and $\tilde{T}$ can be found in [26]. Then the frequency scaling for the propagation factor is

$$
\tilde{F}\left(k_{\rho}, z, z^{\prime}\right) \sim O\left(\omega^{0}\right) .
$$

Finally the frequency scaling for the matrix element in (21)-(25) is

$$
\begin{aligned}
& \overline{\mathbf{Z}}^{s s} \sim O\left(\omega^{0}\right), \overline{\mathbf{Z}}^{z z} \sim O\left(\omega^{0}\right), \overline{\mathbf{Z}}^{z_{1}} \sim O\left(\omega^{0}\right), \\
& \overline{\mathbf{Z}}^{z_{2}} \sim O\left(\omega^{0}\right), \overline{\mathbf{Z}}^{\phi} \sim O\left(\omega^{-2}\right) .
\end{aligned}
$$

We can separate the matrix into two parts according to the frequency scaling

$$
\begin{aligned}
\overline{\mathbf{A}} & =\mu_{m r}\left[\overline{\mathbf{Z}}^{s s}+\overline{\mathbf{Z}}^{z z}+\overline{\mathbf{Z}}^{z_{1}}+\overline{\mathbf{Z}}^{z_{2}}\right] \\
\overline{\mathbf{S}} & =-\frac{k_{n m}^{2}}{\epsilon_{n r}} \overline{\mathbf{Z}}^{\phi} .
\end{aligned}
$$

So that we can have

$$
\overline{\mathbf{Z}}=i k_{0} \eta_{0} \overline{\mathbf{A}}+\frac{\eta_{0}}{i k_{0}} \overline{\mathbf{S}}
$$

where

$$
\overline{\mathbf{A}} \sim O\left(\omega^{0}\right), \overline{\mathbf{S}} \sim O\left(\omega^{0}\right)
$$

Equation (37) has the same form as (8) in free space, which allows us to augment the EFIE in a similar fashion as in (17).

Since most material is non-magnetic, namely $\mu_{i}=\mu_{j}$, we discuss this situation separately. The frequency scaling of the 
Fresnel reflection coefficient for TE wave is an high order term of frequency,

$$
R_{i, j}^{T E}=\frac{\mu_{j} k_{i z}-\mu_{i} k_{j z}}{\mu_{j} k_{i z}+\mu_{i} k_{j z}}=\frac{k_{i z}-k_{j z}}{k_{i z}+k_{j z}} \sim O\left(\omega^{2}\right) .
$$

Then we have

$$
\tilde{R}_{i, j}^{T E} \sim O\left(\omega^{2}\right)
$$

However, we still have

$$
\tilde{M}_{m}^{T E} \sim O\left(\omega^{0}\right), \tilde{T}_{m n}^{T E} \sim O\left(\omega^{0}\right) .
$$

Notice the fact that except for the $\overline{\mathbf{Z}}^{s s}$, other four terms in (22)-(25) consist of TE as well as TM wave, and the TM part is still on $O\left(\omega^{0}\right)$. This means that the frequency scaling for these four terms in (34) are still valid. By careful dimensional analysis, we can show that even for $\overline{\mathbf{Z}}^{s s}$, the leading order term is still $O\left(\omega^{0}\right)$. In a word, for non-magnetic material, (34)-(38) are also valid.

Dielectric loss and conductor loss can be introduced to alleviate the low frequency breakdown in free space [21]. For a structure embedded in a layered medium, if we introduce dielectric loss to each layer, since the equivalent permittivity is

$$
\tilde{\epsilon}_{r}=\epsilon_{r}+\frac{i \sigma}{\omega \epsilon_{0}}
$$

the frequency scaling of the $Z_{\phi}$ becomes

$$
\overline{\mathbf{Z}}^{\phi} \sim O\left(\omega^{-1}\right)
$$

so the scalar potential matrix becomes

$$
\overline{\mathbf{S}} \sim O\left(\omega^{1}\right) .
$$

The A-EFIE can then take the alternative form

$$
\left[\begin{array}{cc}
\overline{\mathbf{A}} & \left(i k_{0}\right)^{-1} \overline{\mathbf{D}}^{T} \cdot \overline{\mathbf{P}} \\
\overline{\mathbf{D}} & -i k_{0} \overline{\mathbf{I}}
\end{array}\right] \cdot\left[\begin{array}{c}
\mathbf{J} \\
c_{0} \boldsymbol{\rho}
\end{array}\right]=\left[\begin{array}{c}
\left(i k_{0} \eta_{0}\right)^{-1} \mathbf{V} \\
\mathbf{0}
\end{array}\right] .
$$

\section{Consistency Validation}

We discuss two extreme cases to analyze the consistency of the A-EFIE for layered medium Green's function. One with homogeneous layers (free space) and the other with perfect electrical conductor (PEC) layer (half space), both of which have closed form Green's functions.

1) Free Space: In free space, the EFIE can be separated into two parts, the vector potential part and the scalar potential part, both of which are scalar problems with scalar Green's function, because of the homogeneity of the medium, shown in (7)-(10). However, in the layered medium, the response of a dipole is polarization dependent. A vertical electric dipole can only generate a TM wave, while a horizontal electric dipole generates TE as well as TM waves. The polarization dependency leads to the difficulty in defining a uniform scalar potential. In the matrix friendly formula, we can see $\overline{\mathbf{Z}}^{s s}$ and $\overline{\mathbf{Z}}^{z z}$ manifest the different response of a horizontal and a vertical dipole in a layered medium. If we asymptotically make the inhomogeneity disappear, namely, make $\epsilon_{m r} \rightarrow 1$ and $\mu_{m r} \rightarrow 1$ for each layer, and applying the Sommerfeld identity

$$
g\left(\mathbf{r}, \mathbf{r}^{\prime}\right)=\frac{i}{4 \pi} \int_{0}^{+\infty} d k_{\rho} \frac{k_{\rho}}{k_{z}} J_{0}\left(k_{\rho} \rho\right) e^{i k_{z}\left|z-z^{\prime}\right|}
$$

the $\overline{\mathbf{Z}}^{s s}$ and $\overline{\mathbf{Z}}^{z z}$ recover the polarization independent vector potential in (9). Similar situation holds for the $\overline{\mathbf{Z}}^{\phi}$, it goes back to the scalar potential part in (10) when removing the inhomogeneity. The physical meanings of the remaining two terms $\overline{\mathbf{Z}}^{z 1}$ and $\overline{\mathbf{Z}}^{z 2}$ are ambiguous due to the lack of exact definition of scalar potential. There is no correspondence in free space, since the TE and TM waves cancel each other when the layered medium degrades into a free space. By appearance, we can interpret it as the cross interaction between charge and the vertical current. According to their same frequency scaling with $\overline{\mathbf{Z}}^{\text {ss }}$ and $\overline{\mathbf{Z}}^{z z}$, we can group them together to obtain the layered medium A-EFIE, as is done in (35).

2) Half Space With PEC Layer: For a half space with a PEC layer, the image method can be applied and the dyadic Green's function can be expressed in a closed form [27]

$$
\overline{\mathbf{G}}\left(\mathbf{r}, \mathbf{r}^{\prime}\right)=\left(\overline{\mathbf{I}}-\frac{1}{k_{0}^{2}} \nabla \nabla^{\prime}\right)\left[g\left(\mathbf{r}, \mathbf{r}^{\prime}\right)-g_{i}\left(\mathbf{r}, \mathbf{r}^{\prime \prime}\right)\right]+2 \hat{z} \hat{z} g_{i}\left(\mathbf{r}, \mathbf{r}^{\prime \prime}\right)
$$

where $g\left(\mathbf{r}, \mathbf{r}^{\prime}\right)$ and $g_{i}\left(\mathbf{r}, \mathbf{r}^{\prime \prime}\right)$ is the free space scalar Green's function with real source point $\mathbf{r}^{\prime}$ and image source point $\mathbf{r}^{\prime \prime}$, with $\overline{\mathbf{A}}$ and $\overline{\mathbf{S}}$ defined as

$$
\begin{aligned}
{[\overline{\mathbf{A}}]_{j i}=} & \left\langle\mathbf{f}_{j}(\mathbf{r}), g\left(\mathbf{r}, \mathbf{r}^{\prime}\right)-g_{i}\left(\mathbf{r}, \mathbf{r}^{\prime \prime}\right), \mathbf{f}_{i}\left(r^{\prime}\right)\right\rangle \\
& +2\left\langle\hat{z} \cdot \mathbf{f}_{j}(\mathbf{r}), g_{i}\left(\mathbf{r}, \mathbf{r}^{\prime \prime}\right), \hat{z} \cdot \mathbf{f}_{i}\left(r^{\prime}\right)\right\rangle \\
{[\overline{\mathbf{S}}]_{j i}=} & \left\langle\nabla \cdot \mathbf{f}_{j}(\mathbf{r}), g\left(\mathbf{r}, \mathbf{r}^{\prime}\right)-g_{i}\left(\mathbf{r}, \mathbf{r}^{\prime \prime}\right), \nabla^{\prime} \cdot \mathbf{f}_{i}\left(\mathbf{r}^{\prime}\right)\right\rangle
\end{aligned}
$$

where we assume the interface is at $z=0$, so $r^{\prime}=\left(x^{\prime}, y^{\prime}, z^{\prime}\right)$, and $\mathbf{r}^{\prime \prime}=\left(x^{\prime}, y^{\prime},-z^{\prime}\right)$. Notice the fact that $R^{T E}=-1$ and $R^{T M}=1$ at the interface in the propagation factor $F\left(k_{\rho}, z, z^{\prime}\right)$, we can reproduce (48) and (49) by our general A-EFIE formula, with the help of Sommerfeld identity.

Although the terms $\overline{\mathbf{Z}}^{z 1}$ and $\overline{\mathbf{Z}}^{z 2}$ cannot be validated by this two cases, we can show their significance by numerical examples, where vertical structure exists in a dielectric layered medium, and the two terms are always there with nonzero value.

\section{Charge Neutrality Issue}

Charge neutrality enforcement is very important in the A-EFIE for low-frequency problems, as stated in [23]. The motivation of enforcing the charge neutrality is because of the rank deficiency of the A-EFIE. For the A-EFIE shown in (17), the upper block is exactly the same as the traditional EFIE except that the scalar potential part is expressed in terms of patch basis and charge is separated as a set of independent unknowns. The lower block constraints the current continuity condition to make the augmented system solvable. However, rank deficiency exists in the A-EFIE matrix, due to the definition of the 
incidence matrix $\overline{\mathbf{D}}$. Here, $k_{0}^{2}$ is an eigenvalue of the A-EFIE and it tends to zero finally when the frequency goes to DC [23]. Usually, the deflation method [28] can be applied to remove the smallest eigenvalue, for example, in the CCIE Formula [20]. Motivated by the basis rearrangement preconditioner in the loop-tree decomposition [6], we can also apply the charge neutrality enforcement to remedy this problem [23]. This is driven by the physical observation of the problem, and can be easily extended to different layered medium problems.

We discuss this issue in the context of layered medium. If it is backed by a conducting ground plane, which is a common situation in the circuit problems, it acts as a "charge bath" and absorbs the extra charge of the structure, so we should distinguish situations whether there is a via connected to the ground in some parts of the structure. As will be shown in the following, the condition number is always bounded when the frequency goes to $\mathrm{DC}$, by properly enforcing the charge neutrality condition.

\section{A. Structures Not Connected to a Ground Plane}

If the structure is not connected to the "charge bath", the total charge is always neutral. This condition shall be enforced when in low frequency domain due to the rank deficiency. By definition of the RWG basis, the matrix $\overline{\mathbf{D}}$ is linearly dependent or singular. It is evident that the lower block sub-matrix of the A-EFIE is rank deficient when the frequency is low $\left(k_{0} \rightarrow 0\right)$. It is necessary to enforce the charge neutrality condition to make the lower block with full rank.

Two transform matrices can be introduced to fulfill the charge neutrality enforcement [23], and the final A-EFIE system becomes:

$$
\left[\begin{array}{cc}
\overline{\mathbf{A}} & \overline{\mathbf{D}}^{T} \cdot \overline{\mathbf{P}} \cdot \overline{\mathbf{B}} \\
\overline{\mathbf{F}} \cdot \overline{\mathbf{D}} & k_{0}^{2} \overline{\mathbf{I}}_{r}
\end{array}\right] \cdot\left[\begin{array}{l}
i k_{0} \mathbf{J} \\
c_{0} \boldsymbol{\rho}_{r}
\end{array}\right]=\left[\begin{array}{c}
\eta_{0}^{-1} \mathbf{V} \\
\mathbf{0}
\end{array}\right]
$$

with the forward and backward transform as

$$
\rho_{r}=\overline{\mathbf{F}} \cdot \boldsymbol{\rho}, \quad \rho=\overline{\mathbf{B}} \cdot \boldsymbol{\rho}_{r}
$$

where $\boldsymbol{\rho}_{r}$ is the reduced charge unknowns and the $\overline{\mathbf{I}}_{r}$ is the reduced identity matrix.

A rectangular loop embedded in a seven-layer medium is shown in Fig. 1, with its layer parameters specified in the figure. The condition numbers versus frequency are demonstrated in Fig. 2. we can see that without charge neutrality enforcement, the condition number grows unboundedly when decreasing the frequency, it increases in the order of $1 / k_{0}^{2}$ because of the right lower block. The eigenvalue distribution of the A-EFIE matrix at $f=1 \mathrm{~Hz}$ is shown in Fig. 3. After the charge neutrality enforcement, the smallest eigenvalue has been removed away from the origin. We can also observe that when frequency increases, however, the lower-right block is an identity matrix scaled by $k_{0}^{2}$, thus the lower block is no longer singular and such enforcement is no longer necessary.

\section{B. Structures Connected to a Ground Plane}

In this case, the charge neutrality condition cannot be guaranteed since the "charge bath" absorbs the extra charge. The incidence matrix $\overline{\mathbf{D}}$ is no longer singular. In this situation, no special
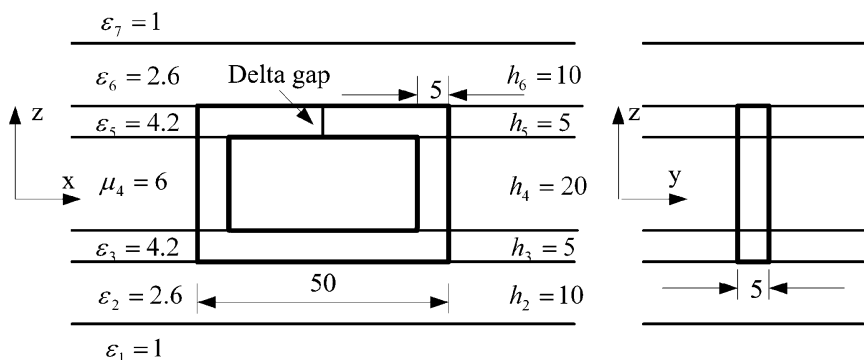

Fig. 1. The geometrical structure of the loop inductor embedded in a sevenlayer medium, unit: $\mathrm{mm}$. The central layer is a magnetic material. A delta gap excitation is applied at the center of the top arm.

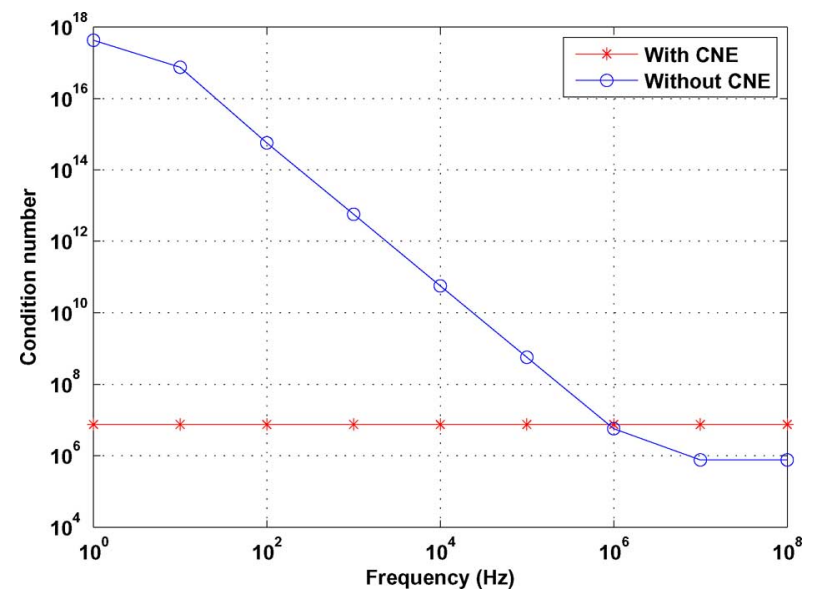

Fig. 2. The condition number versus frequency for the rectangular loop. The condition number is unbounded when decreasing the frequency. Charge neutrality enforcement $(\mathrm{CNE})$ makes the condition number constant.

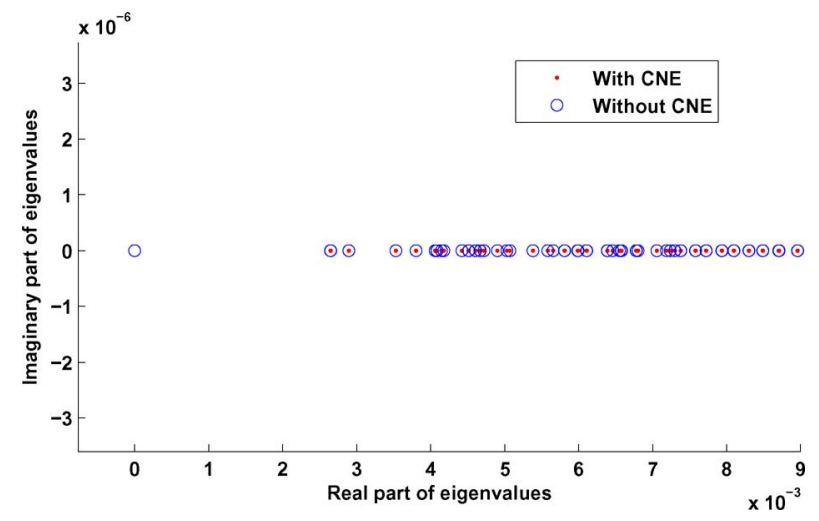

Fig. 3. The eigenvalue distribution for the rectangular loop at $1 \mathrm{~Hz}$. The smallest eigenvalue is removed away from the origin after the charge neutrality enforcement (CNE).

treatment is needed since the A-EFIE system is with full rank. For a half rectangular loop connected to the ground plane shown in Fig. 4, the condition number versus frequency is shown in Fig. 5. Since the A-FEIE matrix is no longer singular because of the ground plane, the condition number remains constant when decreasing the frequency without any special treatment.

Here, the forward and backward transform matrices become the identity matrix in this situation

$$
\overline{\mathbf{F}}=\overline{\mathbf{B}}=\overline{\mathbf{I}} \text {. }
$$



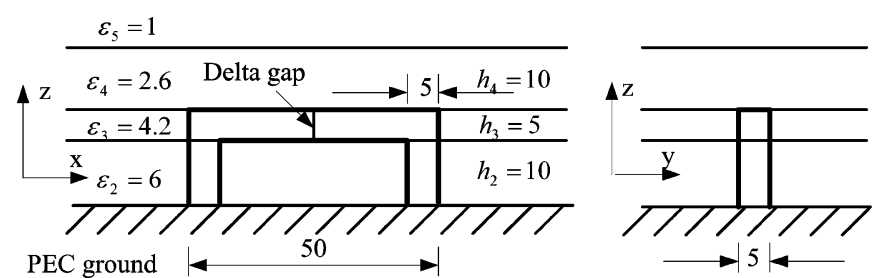

Fig. 4. The geometrical model of the half loop embedded in a five-layer medium (including the PEC layer), unit: $\mathrm{mm}$. A delta gap excitation is applied at the center of the top arm.

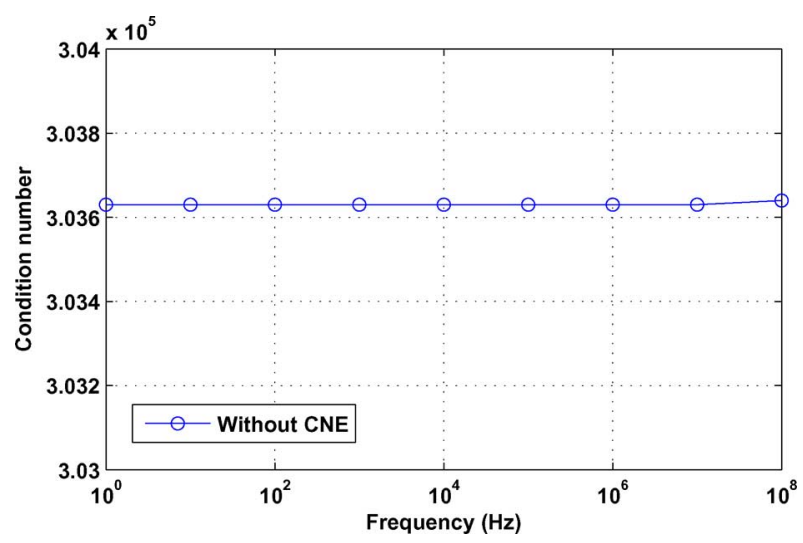

Fig. 5. The condition number versus frequency for the half loop. Since it is connected to the ground plane, charge neutrality cannot be guaranteed. The condition number is bounded when decreasing the frequency without any special treatment.

This observation can be used as a guideline when dealing with complex structures. For a structure with $s$ independent surfaces, each with $p_{k}$ triangular patches, $i_{k}$ inner edges and $g_{k}$ ground edges, $k=1,2 \ldots s$. If there are $m$ surfaces connected to the ground plane, then the total number of unknowns is

$$
N=\sum_{k=1}^{s}\left(i_{k}+g_{k}+p_{k}\right)-m .
$$

One should note that though the number of unknowns in A-EFIE increases much compared to the loop-tree decomposition, where the number of unknowns is the same as the number of RWG basis, the memory requirement increases marginally since all transformation matrices such as $\overline{\mathbf{D}}, \overline{\mathbf{F}}$ and $\overline{\mathbf{B}}$ are all sparse and consume marginal memory when iterative solver is applied.

\section{Electrostatic Limit}

In the electrostatic limit, the electric potential is expressed by the following boundary value problem [3]

$$
\begin{aligned}
& \nabla^{2} \phi=s(\mathbf{r}) \\
& \phi(\mathbf{r})=0 \quad \mathbf{r} \in S
\end{aligned}
$$

and the integral equation becomes

$$
\left\langle G_{s}\left(\mathbf{r}, \mathbf{r}^{\prime}\right), \sigma\left(\mathbf{r}^{\prime}\right)\right\rangle=-\epsilon_{0} \phi_{0}(\mathbf{r}) \quad \mathbf{r} \in S
$$

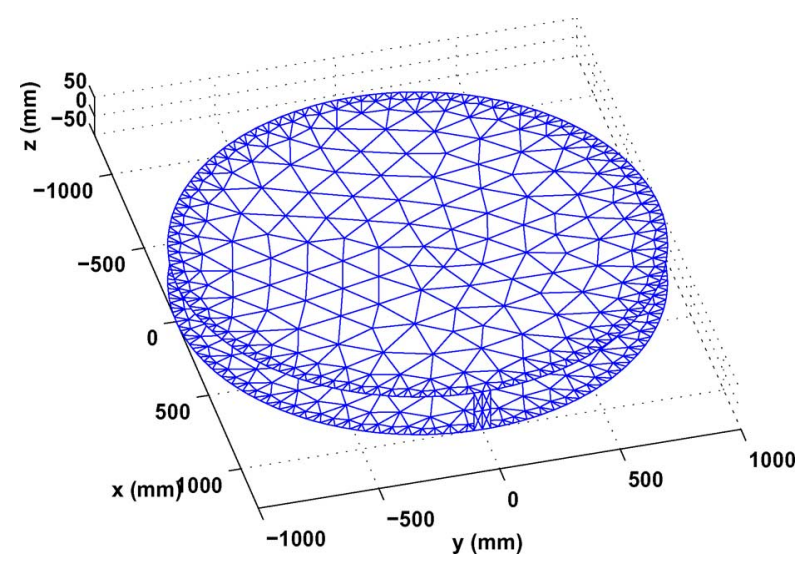

Fig. 6. The geometrical model of the circular parallel plate capacitor, with a dielectric layer $\left(\epsilon_{r}=2.65\right)$ inserted in between. A delta gap is applied at the edge. The mesh is refined to capture the fringing effect.

where $\sigma$ is the surface charge density, $G_{s}\left(\mathbf{r}, \mathbf{r}^{\prime}\right)$ is the static layered medium Green's function [30] and $\phi_{0}$ is the potential generated by the source $s(\mathbf{r})$. In this limit, a typical problem is the parallel plate capacitor, shown in Fig. 6. Here we connect the two plates with a narrow strip so that we can apply the electrodynamic analysis.

In this limit, the A-EIFE suffers from an inaccuracy problem, even though the matrix is nonsingular, because the current is a higher order term in frequency. To capture current accurate to arbitrary order, the perturbation method should be applied [29]. We will show that the charge is always stable and describes the electrostatic physics. In DC, the A-EFIE becomes

$$
\left[\begin{array}{cc}
\overline{\mathbf{A}}_{s} & \overline{\mathbf{D}}^{T} \cdot \overline{\mathbf{P}}_{s} \cdot \overline{\mathbf{B}} \\
\overline{\mathbf{F}} \cdot \overline{\mathbf{D}} & 0
\end{array}\right] \cdot\left[\begin{array}{c}
i k_{0} \mathbf{J} \\
c_{0} \boldsymbol{\rho}_{r}
\end{array}\right]=\left[\begin{array}{c}
\eta_{0}^{-1} \mathbf{V} \\
\mathbf{0}
\end{array}\right]
$$

where $\overline{\mathbf{A}}_{s}$ and $\overline{\mathbf{P}}_{s}$ are matrix evaluated at $k_{0}=0$. For this problem, the current $\mathbf{J}$ disappears, while the charge remains constant,

$$
\mathbf{J} \rightarrow 0, \rho \rightarrow c .
$$

Since the matrix is still full rank, we have unique solution. However, the current is no longer correct due to the finite numerical precision. If we discard the $i k_{0} \mathbf{J}$ term manually, we have

$$
\overline{\mathbf{D}}^{T} \cdot \overline{\mathbf{P}}_{s} \cdot \overline{\mathbf{B}} \cdot \rho_{r}=\epsilon_{0} \mathbf{V}
$$

It is shown that the electrostatic information is included in the A-EFIE. In free space, it is straightforward to check that the static Green's function in (56) and (59) is the same one

$$
G_{s}\left(\mathbf{r}, \mathbf{r}^{\prime}\right)=\frac{1}{4 \pi\left|\mathbf{r}-\mathbf{r}^{\prime}\right|}
$$

In a layered medium, we will show the static form of the general matrix-friendly Green's function in $\overline{\mathbf{P}}_{s}$ in the appendix, 


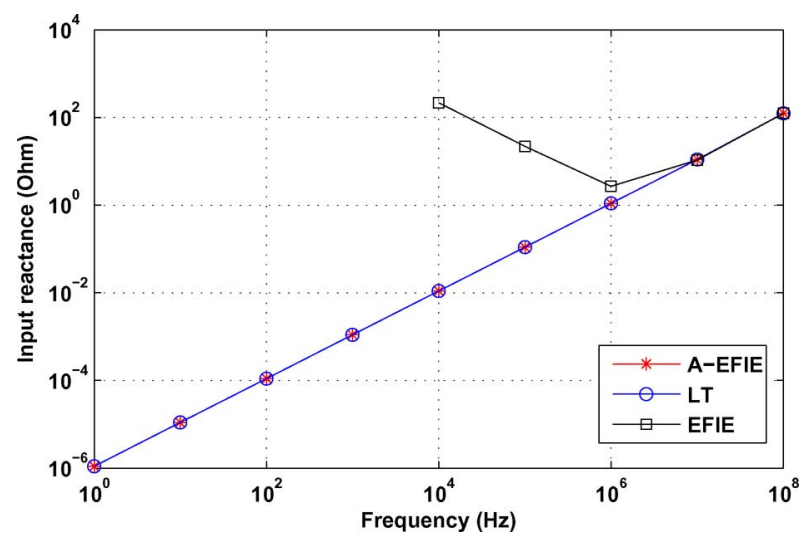

Fig. 7. The input reactance of the rectangular loop. A-EFIE agrees well with the loop-tree (LT) decomposition, while the traditional EFIE breaks down quickly when decreasing the frequency.

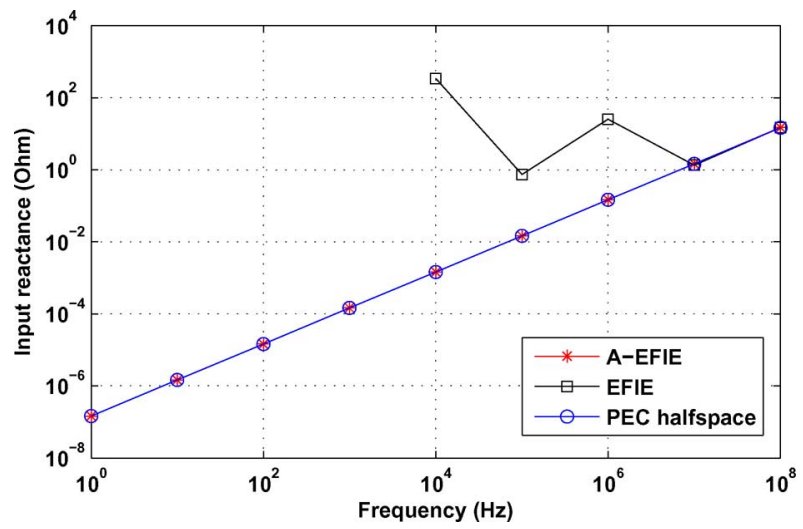

Fig. 8. The input reactance of the half loop. A-EFIE maintains the scale invariance very well while the traditional EFIE breaks down quickly when decreasing the frequency. Since the non-magnetic dielectric is transparent to the inductor, a PEC half space model is applied to validate the results.

which agrees with that of [30] applied in the electrostatic analysis except for minor differences such as the layer index, constant $1 / 4 \pi \epsilon_{0}$ and the sign definition in the Fresnel reflection coefficient. Numerical results will be given to further validate our statement in the next section.

\section{NUMERICAL RESULTS}

Several numerical results are presented in this section. The input reactance of the rectangular loop shown in Fig. 1 is calculated, where the results are compared with the loop-tree (LT) decomposition and traditional EFIE in Fig. 7. The EFIE breaks down quickly when the frequency decreases, while the A-EFIE is very stable and agrees very well with the loop-tree decomposition. We also calculate the input reactance of the half rectangular loop mentioned above. Because it is connected to a conducting ground plane, the current can flow along this half loop. The input reactance is shown in Fig. 8 compared with traditional EFIE. Similar phenomenon can be observed. Since non-magnetic dielectric is transparent to the loop inductor, the PEC half space model can be used to validate the result, which is also shown in Fig. 8. Good agreement with the A-EFIE for general layered medium can be observed. Finally, a circular parallel plate capacitor with radius of unit length $(a=1 \mathrm{~m})$ is shown in Fig. 6 with

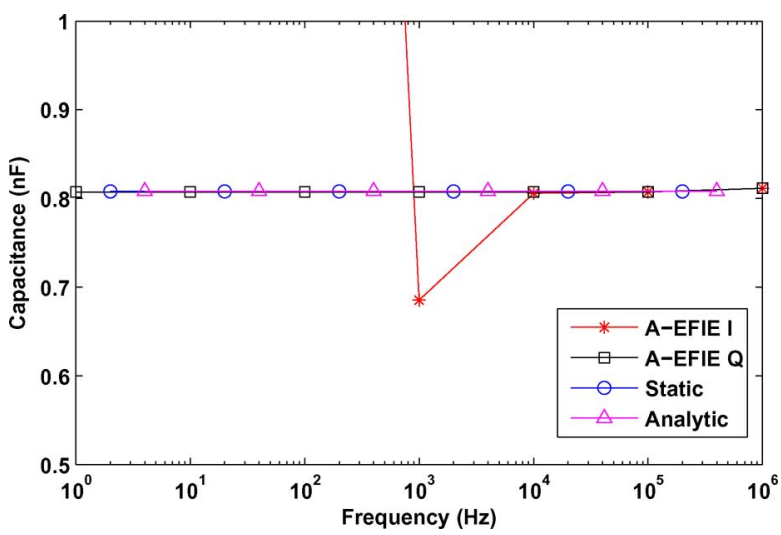

Fig. 9. The capacitance of the circular parallel plate capacitor. A-EFIE I represents the capacitance extracted from current, while A-EFIE Q means the capacitance extracted from charge. The A-EFIE current suffers from an inaccuracy problem while the A-EFIE charge is stable. The result agrees with the static solver. Both are further validated by the analytic solution. When the frequency is below $1 \mathrm{MHz}$, the relative error of A-EFIE $\mathrm{Q}$ is around $0.1 \%$.

a dielectric layer inserted in between $\left(\epsilon_{r}=2.65\right)$. The distance is set to be $d / a=0.1$. The capacitance extracted from current and charge and static solver are shown in Fig. 9. The current suffers from an inaccuracy problem, as mentioned in last section, while the charge is accurate and agrees with the static analysis. The analytic result from asymptotic expansion [31] is also shown to validate the numerical results. In this example, when the frequency is below $1 \mathrm{MHz}$, the relative error of the A-EFIE with charge information is around $0.1 \%$. If the frequency is increased, wave physics begins to play a role and the parallel plate is no longer a pure capacitor.

\section{CONCLUSION}

An augmented EFIE for layered medium Green's function is developed in this paper. The frequency scaling is analyzed for both lossless and lossy media. The rank deficiency of the A-EFIE in layered media depends on if the charge neutrality condition is satisfied. For independent structures, the enforcement is necessary in the low frequency regime, while at midfrequencies, such implementation is no longer necessary. For structures connected to the ground, the A-EFIE matrix is full rank, and no special treatment is needed. The electrostatic limit is analyzed and compared with the static formulation. Several numerical results are presented to validate this method.

\section{APPENDIX}

\section{Electrostatic LAYERED MEdiUM GREen’s FunCtion}

In the electrostatic limit, the layered medium Green's function shown in [30] can be derived from our general matrixfriendly formulation

$$
\begin{aligned}
G_{s}\left(\mathbf{r}, \mathbf{r}^{\prime}\right) & =-\frac{1}{\epsilon_{n r}} \partial_{z} \partial_{z^{\prime}} g^{T M}\left(\mathbf{r}, \mathbf{r}^{\prime}\right) \\
& =\frac{1}{4 \pi \epsilon_{n r}} \sum_{i} \int_{0}^{+\infty} d \lambda J_{0}(\lambda \rho) e^{-\lambda Z_{i}} \Omega_{i}
\end{aligned}
$$

where the Green's function is described by several images with weight $\Omega_{i}$ and distance $Z_{i}$. 
$m=n:$ When $m=n$, there are one primary term and four image terms

$$
\begin{aligned}
& \Omega_{0}=1, Z_{0}=\left|z-z^{\prime}\right| \\
& \Omega_{1}=-\tilde{R}_{m, m-1} \tilde{M}_{m}, Z_{1}=-2 d_{m}+z^{\prime}+z \\
& \Omega_{2}=-\tilde{R}_{m, m+1} \tilde{M}_{m}, Z_{2}=2 d_{m+1}-z^{\prime}-z \\
& \Omega_{3}=\tilde{R}_{m, m+1} \tilde{R}_{m, m-1} \tilde{M}_{m}, Z_{3}=2 h_{m}+z^{\prime}-z \\
& \Omega_{4}=\tilde{R}_{m, m+1} \tilde{R}_{m, m-1} \tilde{M}_{m}, Z_{4}=2 h_{m}-z^{\prime}+z .
\end{aligned}
$$

$m<n$ : When $m<n$, there are four image terms

$\Omega_{1}=\tilde{T}_{m n}^{+} \tilde{M}_{m}, Z_{1}=-z^{\prime}+z$

$\Omega_{2}=-\tilde{R}_{n, n+1} \tilde{T}_{m n}^{+} \tilde{M}_{m}, Z_{2}=2 d_{n+1}-z^{\prime}-z$

$\Omega_{3}=-\tilde{R}_{m, m-1} \tilde{T}_{m n}^{+} \tilde{M}_{m}, Z_{3}=-2 d_{m}+z^{\prime}+z$

$\Omega_{4}=\tilde{R}_{m, m-1} \tilde{R}_{n, n+1} \tilde{T}_{m n}^{+} \tilde{M}_{m}, Z_{4}=-2 d_{m}+2 d_{n+1}+z^{\prime}-z$.

$m>n:$ When $m>n$, there are also four image terms

$$
\begin{aligned}
& \Omega_{1}=\tilde{T}_{m n}^{-} \tilde{M}_{m}, Z_{1}=z^{\prime}-z \\
& \Omega_{2}=-\tilde{R}_{n, n-1} \tilde{T}_{m n}^{-} \tilde{M}_{m}, Z_{2}=-2 d_{n}+z^{\prime}+z \\
& \Omega_{3}=-\tilde{R}_{m, m+1} \tilde{T}_{m n}^{-} \tilde{M}_{m}, Z_{3}=2 d_{m+1}-z^{\prime}-z \\
& \Omega_{4}=\tilde{R}_{m, m+1} \tilde{R}_{n, n-1} \tilde{T}_{m n}^{-} \tilde{M}_{m}, Z_{4}=2 d_{m+1}-2 d_{n}-z^{\prime}+z
\end{aligned}
$$

where

$$
\begin{aligned}
\tilde{R}_{i, i+1} & =\frac{R_{i, i+1}+\tilde{R}_{i+1, i+2} e^{-2 \lambda h_{i+1}}}{1+R_{i, i+1} \tilde{R}_{i+1, i+2} e^{-2 \lambda h_{i+1}}} \\
\tilde{R}_{i, i-1} & =\frac{R_{i, i-1}+\tilde{R}_{i-1, i-2} e^{-2 \lambda h_{i-1}}}{1+R_{i, i-1} \tilde{R}_{i-1, i-2} e^{-2 \lambda h_{i-1}}} \\
\tilde{M}_{m} & =\frac{1}{1-\tilde{R}_{m, m-1} \tilde{R}_{m, m+1} e^{-2 \lambda h_{m}}} \\
\tilde{T}_{m n}^{+} & =\prod_{j=m}^{n-1} \frac{T_{j, j+1}}{1-R_{j+1, j} \tilde{R}_{j+1, j+2} e^{-2 \lambda h_{j+1}}} \\
\tilde{T}_{m n}^{-} & =\prod_{j=n}^{m-1} \frac{T_{j+1, j}}{1-R_{j, j+1} \tilde{R}_{j, j-1} e^{-2 \lambda h_{j}}} \\
h_{i} & =d_{i+1}-d_{i} .
\end{aligned}
$$

\section{REFERENCES}

[1] R. H. Harrington, Field Computation by Moment Methods. New York: IEEE Press, 1993

[2] S. M. Rao, D. R. Wilton, and A. W. Glisson, "Electromagnetic scattering by surface of arbitrary shape," IEEE Trans. Antennas Propag., vol. 30, pp. 409-418, May 1982.

[3] W. C. Chew, M. S. Tong, and B. Hu, Integral Equations for Electromagnetic and Elastic Waves. London, U.K.: Morgan \& Claypool, 2009.
[4] D. R. Wilton and A. W. Glisson, "On improving the electric field integral equation at low frequencies," in URSI Radio Science Meeting Dig., Los Angeles, CA, Jun. 1981, pp. 24-24.

[5] G. Vecchi, "Loop-star decomposition of basis functions in the discretization of the EFIE," IEEE Trans. Antennas Propag., vol. 47, pp. 339-346, Feb. 1999.

[6] J. S. Zhao and W. C. Chew, "Integral equation solution of Maxwell's equations from zero frequency to microwave frequencies," IEEE Trans. Antennas Propag., vol. 48, pp. 1635-1645, Oct. 2000.

[7] J. F. Lee, R. Lee, and R. J. Burkholder, "Loop-star basis functions and a robust preconditioiner for EFIE scattering problems," IEEE Trans. Antennas Propag., vol. 51, pp. 1855-1863, Aug. 2003.

[8] Q. Chen and D. R. Wilton, "Electromagnetic scattering by three-dimensional arbitrary complex material/conducting bodies," in Proc. Antennas Propag. Soc. Int. Symp., 1990, pp. 590-593.

[9] A. Buffa and S. Christiansen, "A dual finite element complex on the barycentric refinement," Math. Comput., vol. 76, pp. 1743-1769, 2007.

[10] F. P. Andriulli, K. Cools, H. Bagei, F. Olyslager, A. Buffa, S. Christiansen, and E. Michielssen, "A multiplicative Calderón preconditioner for the electric field integral equation," IEEE Trans. Antennas Propag., vol. 56, no. 8, pp. 2398-2412, Aug. 2008.

[11] M. B. Stephanson and J. F. Lee, "Preconditioned electric field integral equation using Calderón identities and dual loop/star basis functions," IEEE Trans. Antennas Propag., vol. 57, pp. 1274-1279, Apr. 2009.

[12] S. Yan, J. M. Jin, and Z. P. Nie, "EFIE Analysis of low-frequency problems with loop-star decomposition and Calderon multiplicative preconditioner," IEEE Trans. Antennas Propag., vol. 58, pp. 857-867, Mar. 2010.

[13] K. A. Michalski and D. Zheng, "Electromagnetic scattering and radiation by surfaces of arbitrary shape in layered media, Part I: Theory," IEEE Trans. Antennas Propag., vol. 38, pp. 335-344, Mar. 1990.

[14] P. Ylä-Oijala, M. Taskinen, and J. Sarvas, "Multilayered media Green's functions for MPIE with general electric and magnetic sources by the Hertz potential approach," Progr. Electromagn. Res. PIER, vol. 33, pp. 141-165, 2001

[15] W. C. Chew, J. L. Xiong, and M. A. Saville, "A matrix-friendly formulation of layered medium Green's function," IEEE Antennas Wireless Propag. Lett., vol. 5, pp. 490-494, 2006.

[16] J. L. Xiong and W. C. Chew, "A newly developed formulation suitable for matrix manipulation of layered medium Greens functions," IEEE Trans. Antennas Propag., vol. 58, pp. 868-875, Mar. 2010.

[17] S. Uckun, T. K. Sarkar, S. M. Rao, and M. Salazar-Palma, "A novel technique for analysis of electromagnetic scattering from microstrip antennas of arbitrary shape," IEEE Trans. Microwave Theory Tech., vol. 45, pp. 485-491, Apr. 1997.

[18] F. Ling, V. I. Okhmatovski, W. Harris, S. McCracken, and A. Dengi, "Large-scale broadband parasitic extraction for fast layout verification of 3-D RF and mixed-signal on-chip structures," IEEE Trans. Microw. Theory Tech., vol. 53, no. 1, pp. 264-273, Jan. 2005.

[19] Y. P. Chen, J. L. Xiong, W. C. Chew, and Z. P. Nie, "Numerical analysis of electrically small structures embedded in a layered medium," Microw. Opt. Tech. Lett., vol. 51, no. 5, pp. 1304-1308, 2009.

[20] M. Taskinen and P. Ylä-Oijala, "Current and charge integral equation formulation," IEEE Trans. Antennas Propag., vol. 54, pp. 58-67, Jan. 2006.

[21] D. Gope, A. Ruehli, and V. Jandhyala, "Solving low-frequency EM-CKT problems using the PEEC method," IEEE Trans. Adv. Packag., vol. 30, pp. 313-320, May 2007.

[22] Z. G. Qian and W. C. Chew, "An augmented EFIE for high speed interconnect analysis," Microw. Opt. Tech. Lett., vol. 50, no. 10, pp. 2658-2662, Oct. 2008.

[23] Z. G. Qian and W. C. Chew, "Fast full-wave surface integral equation solver for multiscale structure modeling," IEEE Trans. Antennas Propag., vol. 57, pp. 3594-3601, Nov. 2009.

[24] M. Benzi, G. H. Golub, and J. Liesen, "Numerical solution of saddle point problems," Acta Numer., vol. 14, pp. 1-137, Apr. 2005.

[25] J. A. Kong, "Electromagnetic field due to dipole antennas over stratified anisotropic media," Geophysics, vol. 38, pp. 985-996, 1972.

[26] W. C. Chew, Waves and Fields in Inhomogeneous Media. New York Van Nostrand Reinhold, 1990. 
[27] C. T. Tai, Dyadic Green's Functions in Electromagnetic Theory. New York: IEEE Press, 1993.

[28] A. Barnard, I. Duck, M. Lynn, and W. Timlake, "The application of electromagnetic theory to electrocardiography II: Numerical solution of the integral equations," Biophys. J., vol. 7, pp. 463-490, Mar. 1967.

[29] Z. G. Qian and W. C. Chew, "Enhanced A-EFIE with perturbation method," IEEE Trans. Antennas Propag., vol. 58, pp. 3256-3264, Oct. 2010.

[30] Y. C. Pan, "Development of the Fast Multipole Mehtod for Stratified Medium," Ph.D. dissertation, University of Illinois, Urbana-Champaign, 2002.

[31] W. C. Chew and J. A. Kong, "Microstrip capacitance for a circular disk through matched asymptotic expansions," SIAM J. Appl. Math., vol. 42, no. 2, pp. 302-317, Apr. 1982.

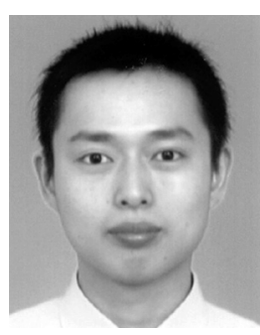

electromagnetics.
Yongpin P. Chen (S'09) was born in Zhejiang, China, in 1981. He received the B.S. and M.S. degrees in microwave engineering from the University of Electronic Science and Technology of China, Chengdu, in 2003 and 2006, respectively. $\mathrm{He}$ is currently working towards the Ph.D. degree in electrical engineering in the University of Hong Kong, Hong Kong, China.

His research interests include waves and fields in inhomogeneous media, integral equation methods and fast algorithms in computational

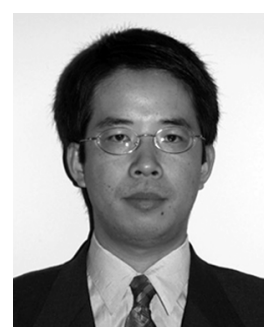

Lijun Jiang (S'01-M'04) received the B.S. degree in electrical engineering from the Beijing University of Aeronautics and Astronautics, China, in 1993, the M.S. degree from Tsinghua University, China, in 1996, and Ph.D. from the University of Illinois at Urbana-Champaign, in 2004.

From 1996 to 1999, he was an application Engineer with Hewlett-Packard. From 2004 to 2009, he was a Postdoctoral Researcher, research staff member, and Senior Engineer at the IBM T.J. Watson Research Center, New York. Since the end of 2009, he has been an Associate Professor with the Department of Electrical and Electronic Engineering, University of Hong Kong. His research interests focus on electromagnetics, IC signal/power integrity, antennas, multidisciplinary EDA solutions, RF and microwave technologies, and high performance computing (HPC), etc.

Prof. Jiang received the IEEE MTT Graduate Fellowship Award in 2003 and the Y.T. Lo Outstanding Research Award in 2004. He is an IEEE Antennas and Propagation Society (AP-S) Member, and a Sigma Xi Associate Member. He was the Semiconductor Research Cooperation (SRC) Industrial Liaison for several academic projects. Since 2009, he has been the SRC Packaging High Frequency Topic TT Chair. He also serves as a Reviewer of IEEE TRANSACTIONS on several topics, and other primary electromagnetics and microwave related journals.

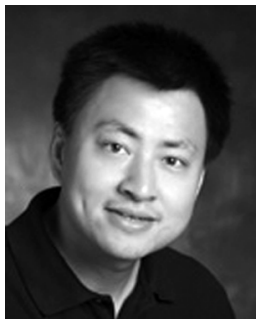

designs.

Dr. Qian was the recipient of the Intel Best Student Paper Award presented at the IEEE 17th Topical Meeting on Electrical Performance of Electronic Packaging (EPEP) in 2008.

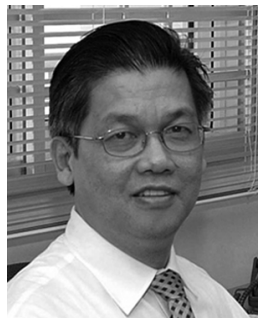

Weng Cho Chew (S'79-M'80-SM'86-F'93) received the B.S. degree in 1976, both the M.S. and Engineer's degrees in 1978, and the Ph.D. degree in 1980, from the Massachusetts Institute of Technology, Cambridge, all in electrical engineering.

He is serving as the Dean of Engineering at The University of Hong Kong. Previously, he was a Professor and the Director of the Center for Computational Electromagnetics and the Electromagnetics Laboratory at the University of Illinois. He was a Founder Professor of the College of Engineering, and previously, the First Y.T. Lo Endowed Chair Professor in the Department of Electrical and Computer Engineering, University of Illinois. Before joining the University of Illinois, he was a Department Manager and a Program Leader at Schlumberger-Doll Research. His research interests are in the areas of waves in inhomogeneous media for various sensing applications, integrated circuits, microstrip antenna applications, and fast algorithms for solving wave scattering and radiation problems. He is the originator several fast algorithms for solving electromagnetics scattering and inverse problems. He has led a research group that has developed parallel codes that solve dense matrix systems with tens of millions of unknowns for the first time for integral equations of scattering. He has authored the book, Waves and Fields in Inhomogeneous Media, coauthored two books, Fast and Efficient Methods in Computational Electromagnetics and Integral Equation Methods for Electromagnetic and Elastic Waves, and authored and coauthored over 300 journal publications, over 400 conference publications and over ten book chapters.

Dr. Chew is a Fellow of the IEEE, OSA, IOP, Electromagnetics Academy, Hong Kong Institute of Engineers (HKIE), and was an NSF Presidential Young Investigator (USA). He received the Schelkunoff Best Paper Award from the IEEE TRANSACTIONS ON ANTENNAS AND PROPAGATION, the IEEE Graduate Teaching Award, UIUC Campus Wide Teaching Award, and IBM Faculty Awards. In 2008, he was elected by the IEEE AP Society to receive the Chen-To Tai Distinguished Educator Award. He served on the IEEE Adcom for the Antennas and Propagation Society as well as the Geoscience and Remote Sensing Society. From 2005 to 2007, he served as an IEEE Distinguished Lecturer. He served as the Cheng Tsang Man Visiting Professor at Nanyang Technological University in Singapore in 2006. In 2002, ISI Citation elected him to the category of Most-Highly Cited Authors (top 0.5\%).He is currently the Editor-in-Chief of JEMWA/PIER journals, and is on the board of directors of the Applied Science Technology Research Institute, Hong Kong. 